\title{
REVISTAACIÓN
}

Revista Educación

ISSN: 0379-7082

ISSN: 2215-2644

revedu@gmail.com

Universidad de Costa Rica

Costa Rica

\section{Identidad profesional de estudiantes de la Facultad de Educación en la Universidad Católica de la Santísima Concepción}

Jaramillo Azema, Cristian; Benoit Ríos, Claudine; Castro Cáceres, Ricardo

Identidad profesional de estudiantes de la Facultad de Educación en la Universidad Católica de la Santísima Concepción

Revista Educación, vol. 44, núm. 1, 2020

Universidad de Costa Rica, Costa Rica

Disponible en: http://www.redalyc.org/articulo.oa? $\mathrm{id}=44060092036$

DOI: https://doi.org/10.15517/revedu.v44i1.37678

Esta obra está bajo una Licencia Creative Commons Atribución-NoComercial-SinDerivar 3.0 Internacional. 


\title{
Identidad profesional de estudiantes de la Facultad de Educación en la Universidad Católica de la Santísima Concepción
}

\author{
The Professional Identity of Student Teachers from the Faculty of Education of the Catholic University of the \\ Santísima Concepción in Chile
}

Cristian Jaramillo Azema

Universidad Católica de la Santísima Concepción, Chile cjaramillo@ucsc.cl

iD http://orcid.org/0000-0002-3824-7872

Claudine Benoit Ríos

Universidad Católica de la Santísima Concepción, Chile

cbenoit@ucsc.cl

(iD http://orcid.org/0000-0002-1791-2212

Ricardo Castro Cáceres

Universidad Católica de la Santísima Concepción, Chile

rcastroc@ucsc.cl

(iD http://orcid.org/0000-0003-2541-777X

DOI: https://doi.org/10.15517/revedu.v44i1.37678

Redalyc: http://www.redalyc.org/articulo.oa?id=44060092036 


\section{INTRODUCCIÓN Y JUSTIFICACIÓN DEL ESTUDIO}

Durante el año 2018 en la Universidad Católica de la Santísima Concepción [UCSC], se desarrolla el Programa de Mejoramiento Institucional USC 1501 de la Universidad Católica de la Santísima Concepción [PMI USC 1501], el cual busca generar "Propuestas de mejoramiento para provocar cambios notables en los procesos de formación inicial docente en la UCSC, con énfasis en el trabajo colaborativo con actores del sistema educativo regional en contextos diversos" (PMI USC 1501, 2015, p. 7). Para abordar este objetivo, se llevaron a cabo varias actividades de investigación, entre las que destaca el presente estudio, el cual visibilizó la importancia de la identidad, tanto institucional como personal del estudiantado, al desarrollar su experiencia en la práctica profesional (estudiantes ad portas de egresar).

El presente documento se basa en los principales hallazgos asociados a la identidad profesional que estos estudiantes en práctica profesional manifestaron en el desarrollo de sus actividades. Es decir, una identidad profesional incipiente, pues están cerrando su etapa de formación y comenzando su integración al campo laboral. La estructura del artículo consiste en la revisión teórica de la identidad profesional, la cual permitió identificar las dimensiones asociadas a la identidad en practicantes profesionales. Posteriormente, se definen los lineamientos tanto cualitativos como cuantitativos del estudio, en cuanto al instrumento, los análisis de datos y la muestra. Finalmente, se exponen los principales hallazgos junto con la proyección y posibles usos de los resultados. Recibir específicamente

Estudios anteriores (Canquiz y Maldonado, 2016; Miranda y Vargas, 2019; Morales, 2016) demuestran la relevancia de identificar aquellos factores que permiten diferenciar a estudiantes, ya sea en cuanto a otras casas de estudio, disciplinas, experiencias y las características de su formación. De esta forma, se desarrolló un estudio de carácter descriptivo, pues se caracterizaron los factores que definen la identidad de estudiantes, mediante su diferenciación con otras casas de estudio.

El objetivo general consistió en caracterizar la identidad profesional de estudiantes en práctica profesional de la Facultad de Educación de la UCSC, para lo cual fue preciso describir los discursos sobre su propia identidad profesional y caracterizar, a través de estos, su relación e influencia en estudiantes del establecimiento donde ejecutan su práctica.

El supuesto de investigación definido para este estudio es: La identidad profesional del practicante de la UCSC le permite desarrollar un vínculo con sus estudiantes, lo cual facilita las actividades pedagógicas con alumnado del establecimiento. En consecuencia, se establece una hipótesis de tipo asociación entre dos factores: Las características de la identidad profesional de las personas practicantes, se traducen en actividades pedagógicas más efectivas con estudiantes de la comunidad escolar, diferenciándose con estudiantes de otras universidades.

Características identitarias que la UCSC busca desarrollar en la formación de estudiantes de la Facultad de Educación

Entendiendo, entonces, la identidad profesional docente, en el contexto del PMI UCSC 1501, como una serie de factores diferenciadores al momento de comparar a estudiantes en práctica profesional de pedagogía de la UCSC, con estudiantado en práctica de otras instituciones de educación superior, se vuelve necesario precisar cuáles son sus características identitarias. En este sentido, el punto de partida para comenzar a dar cuenta del objetivo de investigación, es identificar aquellos elementos diferenciadores que la Universidad busca deliberadamente transmitir a sus estudiantes y, relacionado con ello, las variables que permitieron evaluar hasta qué punto la Universidad ha logrado transmitir dichos elementos.

El perfil de egreso de estudiantes de la Facultad, evidenciado en los informes de autoevaluación, consigna que "Su inspiración cristiana le permite incluir en su búsqueda la dimensión moral, espiritual y religiosa, y valorar las conquistas de la ciencia y la tecnología en la perspectiva total de la persona humana" (Modelo Educativo de la Universidad Católica de la Santísima Concepción, 2017, p. 5). Define, a su vez, la identidad que la Universidad busca transmitir a su alumnado, pues busca diferenciar a estudiantes de pedagogía de 
la UCSC con otras instituciones. Siguiendo esta línea argumental, dicha identidad que se busca transmitir se complementa con las competencias profesionales que, también, se destacan en su perfil de egreso, el cual tiene como fundamento las orientaciones acerca de la misión y la tarea del equipo docente declaradas en los Estándares de Desempeño para la Formación Inicial Docente y en el Marco para la Buena Enseñanza, del Ministerio de Educación de Chile.

Si bien es cierto que la UCSC busca transmitir tanto un sello o identidad institucional, esta no necesariamente se encuentra en la transmisión de las competencias profesionales definidas por el Ministerio de Educación, puesto que todas las facultades de educación de las universidades buscan cumplir con estas competencias profesionales. El factor diferenciador que se propone se fundamenta, entonces, en el sello institucional entendido como principios y valores que distinguen a la UCSC de otras instituciones.

Estos principios y valores que la Facultad de Educación busca fomentar en estudiantes y egresados se expresan en el Programa de Mejoramiento Institucional UCSC 1501,2015, en el que la $i d e n t i d a d$ se propone desde los siguientes focos y valores: a) Formación integral de personas; b) Respeto por la dignidad de la persona humana; c) Sello institucional al servicio de la comunidad; d) Competencias desarrolladas en el proceso de formación. De esta forma, en términos metodológicos, la identidad profesional de estudiantes de la Facultad de Educación de la UCSC es susceptible de ser estudiada desde la percepción que desarrollan sobre su formación profesional.

\section{MARCO CONCEPTUAL}

\section{Identidad profesional y su construcción}

La identidad profesional es un concepto que se asocia a un proceso dinámico y complejo en el cual interactúa la subjetividad personal (la historia personal) y la subjetividad social (pertenencias y construcciones colectivas). Este es el punto base con el que se puede entender la identidad profesional (Cervantes y Gutiérrez, 2013; González, 2017) no obstante, de forma complementaria, se puede concebir como una "identización o consideración de elementos de diferenciación de naturaleza biográfica o personal” (Galaz, 2011, p. 91). Es decir, la identidad profesional se define por: a) las características específicas que posee la identidad de un individuo y una colectividad y b) por el cómo dichas características diferencian y reconocen a un individuo de otro y una colectividad de otra.

El visibilizar las características de la identidad de un grupo o persona implica un desafío, puesto que la identidad es dinámica y cambiante; sin embargo, la relevancia de caracterizar esta identidad radica en que, al hacerlo, es posible identificar discursos de los profesionales sobre sus competencias, metas y valores (De Laurentis, 2015). En síntesis, los estudios sobre la identidad pueden precisar la percepción de sí mismos, en el ámbito individual y el reconocimiento, en el ámbito social/colectivo (Beijard, Meijer y Verloop, 2004; Cattonar, 2001).

Dado que la identidad es un fenómeno complejo y dinámico, es necesario profundizar en el aspecto dinámico de la identidad como fenómeno a estudiar (Camacho, Rojas y Hernández, 2019; Trainer, 2013). En este sentido, existen dos tipos de identidades permanentemente relacionadas entre sí: a) identidades sustanciales que se construyen y se mantienen en el tiempo con independencia del contexto, incluso pueden llegar a reafirmar sus características identitarias ante un proceso de cambio de su contexto; b) identidades situacionales, a la inversa de la anterior, son identidades que poseen componentes transitorios, que varían bajo la influencia de los contextos donde los grupos o individuos socializan (Barberousse y Vargas, 2016).

En términos de la identidad profesional asociada al trabajo docente, la literatura permite reconocer dos tipos de identidad: a) las identidades profesionales asociadas a los procesos de enseñanza, donde cobran gran relevancia aspectos vocacionales y expectativas personales del y la docente y $\mathrm{b}$ ) las identidades profesionales 
flexibles, asociadas a la influencia del contexto donde se desarrolla el trabajo profesional docente (Cattonar, 2001; Tardif, 2004).

En síntesis, se puede establecer que la identidad profesional del cuerpo docente es una construcción que se desarrolla a lo largo de toda la vida del profesorado, en sucesivas etapas y eventos complejos de clasificar, y que la imagen o concepto que cada docente construye de sí como profesional, "reúne dimensiones afectivas y cognitivas, personales y sociales en torno a una constante proyección al pasado y al futuro" (Galaz, 2011, p. 1992).

Considerando, entonces, que la identidad profesional se construye constantemente a lo largo del tiempo, ella es abordada desde la influencia del estudiantado en práctica en la comunidad escolar a la cual pertenece. Esto según la bibliografía existente también resulta fundamental, pues varios autores (Kronenberg y Ramugondo, 2013; Lizana, 2009; Schick, Galaz y Urrutia, 2015) acentúan la importancia, en los estudios sobre identidad profesional docente, en el impacto que estas identidades tienen en comunidades escolares: "cada actividad política de la vida diaria, desde la sala de clases, la relación con sus colegas, como inclusive la actividad gremial de la profesión docente, tendrán un auto-eco-impacto social relacionado con esta propia identidad" (Schick, Galaz y Urrutia, 2015, p. 807). En consecuencia, la influencia en la comunidad escolar se considera una dimensión esencial para este estudio.

A continuación, se presentan las dimensiones identificadas en la revisión bibliográfica efectuada para abordar la identidad profesional, específicamente en cuanto a su relación con estudiantes de los establecimientos (Czop, 2008; Gohier y Alin, 2000; Jiménez, 2015; Maroy, 2001; Vonck, 1995; Woods, 2002).

- Rendimiento de estudiantes: se entenderá operacionalmente como la percepción del estudiantado en práctica respecto del desempeño académico de estudiantes del establecimiento.

- Las características que la institución de formación superior ${ }^{1}$ busca generar en sus egresados de pedagogía: se concebirá operacionalmente como las características que la UCSC busca transmitir a estudiantes de pedagogía, basados en el plan estratégico institucional 2017.

- La interacción entre estudiantes de la Facultad de Educación UCSC y los actores de la comunidad escolar: se entenderá operacionalmente como la valoración que los actores de la comunidad escolar proyectan sobre el y la estudiante en práctica de la UCSC. Dicha valoración se levantará a partir de los discursos de estudiantes en práctica.

\section{Metodología}

Esta investigación se define de tipo cuantitativa; sin embargo, es necesario precisar que se complementa con datos cualitativos para la profundización del análisis de acuerdo al objetivo planteado. La importancia de abordar la identidad profesional con ambos enfoques, para el mismo objeto de estudio, radica en la diversidad de dimensiones identificadas en la revisión conceptual, asociada a la identidad profesional, lo cual permite obtener una visión integral, donde los resultados convergen y aumentan la precisión de los análisis.

Por lo tanto, en el levantamiento de información y en el análisis de datos, fue desarrollada la estrategia de integración multimétodo combinación, cuyo objetivo según Blanco y Pirela (2016) es establecer un método principal de investigación, complementado por un segundo método, abordando de esta manera el mismo objeto de estudio con dos enfoques. De esta manera fue posible fortalecer la validez y legitimidad de la investigación, complementando las fortalezas y debilidades del método principal, con las ventajas del método complementario.

Específicamente, se generó un instrumento con características cuantitativas, pero complementado con preguntas abiertas de tipo cualitativas, para abordar las dimensiones de la identidad profesional; dicho esto, los datos obtenidos a partir de los instrumentos cuantitativos se consideran, en términos de análisis, 
los resultados que permiten responder a los objetivos de investigación, pues a partir de ellos fue posible caracterizar la identidad profesional de la muestra estudiada. Por otro lado, los datos cualitativos cumplen un rol de complementariedad respecto de los primeros. Esto ocurre porque los datos cualitativos y cuantitativos poseen características principalmente descriptivas, pero los primeros expresan asociaciones implícitas de tipo causa-efecto, que los acerca más a un tipo de dato explicativo, expresando de esta manera, la relevancia de la complementariedad de ambos métodos. El instrumento fue aplicado de manera presencial, desarrollando previamente una prueba piloto con estudiantes en práctica (no profesional), llegando a 59 casos; de este modo, fue posible evaluar la pertinencia, significancia y redacción del instrumento.

\section{Elementos transversales de los paradigmas cualitativo y cuantitativo}

Este estudio es de tipo descriptivo (Vieytes, 2004), puesto que se caracterizó la identidad profesional del y la estudiante en práctica de la Facultad de Educación de la UCSC y su relación con estudiantes del establecimiento donde llevan a cabo su práctica, desarrollando para ello un instrumento con un carácter descriptivo de la realidad que se está observando y midiendo, sin intervenir en ella (Bericat, 1998; Vieytes, 2004). Por su parte, las actividades de investigación son de tipo transversal, pues las propuestas formuladas buscan recolectar información en un mismo periodo de tiempo.

Las actividades de investigación desarrolladas pertenecen al tipo aplicadas (Vieytes, 2004), pues se enfocan en consolidar el conocimiento existente sobre la identidad profesional de un grupo específico de estudiantes de una casa de estudios. Las actividades propuestas se basan, principalmente, en investigaciones primarias, pues se efectuó un levantamiento de información como primera fuente de datos.

El instrumento diseñado para levantar los datos, consistió en un cuestionario dotado de preguntas abiertas (de carácter cualitativo) y preguntas con alternativas y de tipo escala (de carácter cuantitativo).

\section{Tratamiento de datos cuantitativos}

Desde el enfoque cuantitativo, se elaboró un análisis estadístico descriptivo, de las preguntas con alternativas y tipo escala del instrumento, recurriendo al análisis de frecuencia absoluta, lo cual permitió visibilizar la distribución y frecuencia de los datos en las dimensiones asociadas a la identidad profesional. Dicho de otra manera, las preguntas de tipo cuantitativo, fueron analizadas bajo los términos de una encuesta, debido a la versatilidad con que este instrumento puede ser adaptado, como lo demuestran en estudios empíricos López y Fachelli (2015), lo cual permitió dar cuenta de las dimensiones asociadas a la identidad profesional. El tratamiento de los datos fue desarrollado con el programa estadístico SPSS y, de forma complementaria, con planillas de cálculo.

\section{Tratamiento de datos cualitativos}

El análisis de preguntas abiertas desde un enfoque cualitativo se considera óptimo respecto de las variables y de los objetivos de investigación, pues este tipo de estudio tiene como núcleo "comprender la intención del acto social, esto es, la estructura de motivaciones que tienen los sujetos, la meta que persiguen, el propósito que orienta su conducta, los valores, sentimientos, creencias que lo dirigen hacia un fin determinado" (Mejía, 2013, p. 280). La recopilación de información focaliza en el sujeto como un ser único, asumiendo que este tiene emociones y percepciones frente a una temática o realidad en particular, que varían del resto.

El corpus textual emergente de las respuestas abiertas fue codificado, en primera instancia, de modo manual y, de forma complementaria, se utilizó un software para la categorización. Los datos cualitativos se analizan 
bajo el enfoque de la Teoría Fundamentada, a través del uso del Método Comparativo Constante (Strauss y Corbin, 2002; Vieytes, 2004).

\section{Características de la muestra}

Respecto de las aplicaciones del instrumento, la Tabla 1 presenta la distribución de practicantes profesionales por carrera, que participaron del estudio durante el primer semestre de 2018, la población total fue de 142 , de los cuales 107 respondieron la encuesta, de manera presencial en todos los casos. Dicha población permite establecer parámetros estadísticos de una muestra probabilística con un 5\% de margen de error y $95 \%$ de confiabilidad. Ello implica la elaboración de análisis estadísticos con seguridad.

En cuanto al análisis cualitativo del instrumento, la saturación de información se logró al momento de aplicar alrededor de 30 instrumentos. Considerando esto, la presentación de resultados establece un diálogo entre los datos cuantitativos y cualitativos.

TABLA 1

Distribución de respuestas según carrera

\begin{tabular}{|lll|}
\hline $\begin{array}{l}\text { Carrera de la Facultad de } \\
\text { Educación }\end{array}$ & $\begin{array}{l}\text { Aplicaciones } \\
\text { del } \\
\text { Instrumento }\end{array}$ & $\begin{array}{l}\text { Practicantes } \\
\text { Profesionales }\end{array}$ \\
\hline $\begin{array}{l}\text { Pedagogía en Educación } \\
\text { Diferencial }\end{array}$ & 50 & 69 \\
\hline $\begin{array}{l}\text { Pedagogía en Educación Básica } \\
\text { Con Menciones }\end{array}$ & 15 & 21 \\
\hline Pedagogía en Educación Física & 30 & 34 \\
\hline $\begin{array}{l}\text { Pedagogía en Educación Media } \\
\text { en Matemática }\end{array}$ & 6 & 10 \\
\hline $\begin{array}{l}\text { Pedagogía en Educación M. en } \\
\text { Lenguaje y Comunicación }\end{array}$ & 2 & 3 \\
\hline $\begin{array}{l}\text { Programa de Formación } \\
\text { Pedagógica }\end{array}$ & 1 & 1 \\
\hline $\begin{array}{l}\text { Pedagogía en Educación Media } \\
\text { en Biología y C.N. }\end{array}$ & 1 & 1 \\
\hline Pedagogía en Inglés & 2 & 3 \\
\hline
\end{tabular}

Fuente: Elaboración propia

La distribución de estudiantes en práctica profesional de las carreras de la Facultad de Educación de la UCSC es desigual, debido a las diferencias en la cantidad de estudiantes por carrera. Esto se considera uno de los límites de esta investigación, puesto que la disparidad de participantes, pude generar la sobrerrepresentación de experiencias de practicantes de carreras con mayor número de estudiantes; por lo tanto, los análisis referirán a los practicantes profesionales de la facultad y no por carrera. Lo anterior, plantea el límite de la profundidad de estos análisis y el posterior uso de los resultados por parte de la Universidad estudiada.

\section{Resultados DE LA INVESTIGACióN}

El estudio sobre identidad profesional de los practicantes profesionales estuvo conformado por tres dimensiones de análisis: a) Influencia de la UCSC en el proceso formativo. b) Capacidades pedagógicas efectivas. c) Valoración del practicante de su proceso formativo. 


\section{Características centrales de la identidad profesional de los practicantes profesionales}

De los análisis cualitativos emergieron dos categorías centrales, que permitieron unir de manera explicativa las tres dimensiones antes mencionadas: a) vocación personal y b) formación desarrollada en la UCSC. Estas categorías se consideran centrales, pues, en conjunto, explican la mayor parte de los resultados estadísticos.

a) La primera categoría central, Vocación Personal, se entiende como las acciones que los practicantes llevan a cabo con estudiantes del establecimiento, las cuales van más allá de su rol docente, preocupándose por aspectos personales del estudiantado. La relevancia de esta categoría radica en que la empatía que los practicantes desarrollan facilita llevar a cabo actividades pedagógicas dentro del aula, tal como se presenta en los siguientes ejemplos:

Lo principal para mí es el respeto, empatía y trato hacia las personas, especialmente, con estudiantes de mi escuela (C. R. Pedagogía Educación Física, comunicación personal, 9 de abril de 2018).

Para los resultados me preocupo de conocer cómo aprenden, qué logros deben tener y cómo apoyarlos para obtenerlos, en ese sentido, siempre trato de evitar que se frustren (V. R. Pedagogía en Lenguaje, comunicación personal, 10 de abril, 2018).

Creo que la relación afectiva y estrategias que uso en clases, dinamizan las sesiones y ayudan a llevar una buena relación con respeto (K. M. Educación Diferencial, comunicación personal, 24 de abril de 2018).

La vocación personal se asocia con la formación integral, el servicio a la comunidad y el respeto por la dignidad de la persona humana, como sellos identitarios que la UCSC busca transmitir a sus estudiantes. Ello se refleja en:

Me motivo personalmente a ser un estudiante integral, aportando a mis estudiantes no solo en lo curricular. Si tienen problemas personales o de otro tipo, tengo la cercanía como para apoyarlos en eso hasta donde pueda (D. Ch. Educación Diferencial, comunicación personal, 24 de abril de 2018).

Considero que el compromiso en el ejercicio de mi práctica docente me permitió tener un buen trato con la comunidad educativa (D. C. Pedagogía en Matemáticas, comunicación personal, 11 de abril de 2018).

Desde primer año nos inculcan el respeto hacia los demás y tratar a estudiantes y al resto de la comunidad como personas y que cada uno de ellos es igualdad e importante (J. A. Educación Diferencial, comunicación personal, 23 de abril de 2018).

Lo anterior se vincula fuertemente con la buena relación que los practicantes manifiestan tener con estudiantes del establecimiento. De hecho, el $98 \%$ de los practicantes (considerando las respuestas buena y muy buena) define su relación con estudiantes del establecimiento donde trabaja de manera positiva. Esta buena relación trae dos consecuencias, sintetizadas en los dos factores que se exponen a continuación.

El primero está ligado a las habilidades pedagógicas que se traducen en clases dinámicas, que facilitan la integración y participación de estudiantes del establecimiento.

A [veces] me involucro con el estudiante, y entrego apoyo, monitoreo y explicación personalizada en el aula común (K. M. Educación Diferencial, comunicación personal, 24 de abril de 2018).

Trato de hacer lo mejor posible en mi trabajo. Uso estrategias entretenidas (C. M. Pedagogía en Educación Básica, comunicación personal, 3 de mayo de 2018).

El segundo está ligado a características del sello institucional de la UCSC, que se desarrollan en el proceso de formación del y la estudiante, específicamente: formación integral y el respeto por la dignidad de la persona humana. Esta idea se relaciona con la cercanía y empatía que la persona practicante genera con cada estudiante del establecimiento. En síntesis, la empatía se explica por dos factores: a) aspectos vocacionales personales del practicante y b) la influencia en la formación de la UCSC. Para efectos prácticos, ello implica, según la percepción de los practicantes, un apoyo al momento de desarrollar sus clases o actividades pedagógicas, lo que permite un desarrollo de actividades pedagógicas más dinámicas y la participación de estudiantes en clases:

La relación afectiva y estrategias que dinamizan las sesiones ayudan a llevar una buena relación con respeto (K. M. Educación Diferencial, comunicación personal, 24 de abril de 2018). 
A la didáctica que entregó mi carrera. Herramientas y recursos para los estudiantes (C. R. Pedagogía Educación Física, comunicación personal, 9 de abril de 2018).

Un aspecto relevante de la vocación personal, manifestado en las respuestas de los practicantes, es que la consideran como un factor independiente, pero, al mismo tiempo, ligado a los procesos de formación que desarrollan en la UCSC. Esta paradoja se explica al considerar la vocación como algo propio, pero influido por actividades formativas en la Universidad.

Creo que el sello profesional dependerá $50 \%$ de cada uno y $50 \%$ de la institución donde estudies (C. G. Pedagogía en Educación Básica, comunicación personal, 6 de mayo de 2018).

Porque la cercanía con los estudiantes que considero me facilita mucho la tarea, se debe a la UCSC y la formación que recibí en casa, esos dos elementos cuentan, si faltara uno creo que se notaría en el grado de cercanía (M. T. Educación Diferencial, comunicación personal, 11 de junio de 2018).

Porque me gusta mucho ser profesora, no solo hay un trabajo de la UCSC, sino que propio, en el ánimo propio, de ejercer la profesión por vocación (R. P. Pedagogía en Lenguaje, comunicación personal, 30 de mayo de 2018).

La citada paradoja se entiende, en definitiva, como la asociación constante entre los dos factores más importantes visibilizados en los análisis cualitativos, pues la vocación y la formación en la universidad se consideran los elementos centrales de la identidad profesional de los practicantes.

b) La segunda categoría central, Formación Desarrollada en la UCSC, consiste tanto en las competencias técnicas que los practicantes adquirieron en su paso por la UCSC, como aspectos identitarios que los distinguen de otros practicantes.

La gran mayoría de los practicantes reconoce, a lo menos, una diferencia con estudiantes de otras instituciones de educación superior (96,3\% reconoció diferencias). Entre ellas, es relevante destacar las siguientes:

a) el compromiso con el aprendizaje de mis estudiantes (58\%)

b) las metodologías participativas y didácticas con las que realizaron las clases (48\%)

c) el trato humano y respetuoso con mis estudiantes (60\%)

d) la dedicación y el esfuerzo por mi trabajo (41\%)

Los resultados demuestran que las diferencias identificadas por los practicantes, se sustentan en la relación entre la vocación personal (diferencias a), c) y d) y la formación desarrollada en la UCSC (diferencia $b$ ). Ejemplos de esta relación entre lo vocacional y la formación son:

En la UCSC nos enseñaron estrategias diversificadas y material didáctico, que me han permitido hacer clases entretenidas y siento que a los chicos eso les gusta y participan mucho (S. A. Educación Diferencial, comunicación personal, 8 de junio de 2018).

En nuestra carrera estamos comprometidos con aportar a los estudiantes en todo lo que se pueda, eso viene de antes, es algo personal, pero en la carrera te motiva a potenciarlo, desde tus compañeros de carrera y también los profesores (M. S. Pedagogía Básica, comunicación, 20 de abril de 2018).

Porque soy una persona respetuosa y me preocupa saludar siempre de buena forma, además, estoy comprometida con lo que hago (M. A. Educación Diferencial, comunicación personal, 28 de junio de 2018).

Lo principal para mí es el respeto, empatía y trato hacia las personas, especialmente con los estudiantes de mi escuela (C. R. Pedagogía Educación Física, comunicación personal, 9 de abril de 2018).

Es importante destacar, sin embargo, que la capacidad para facilitar el aprendizaje de las y los estudiantes es la diferencia que menos veces fue destacada (33\%). Esto parece paradójico, pues, al momento de preguntar directamente mediante una escala Likert, si el practicante logró aportar significativamente en el rendimiento académico de estudiantes con los cuales trabajó, el 74\% (sumando de acuerdo y muy de acuerdo) consideró que sí influyó significativamente en los rendimientos académicos.

Esta aparente contradicción es interesante de observar en términos del análisis cualitativo, dado que las personas practicantes reconocen dos posturas al respecto. La primera consiste en el reconocimiento de que 
no han podido posicionarse como un factor que marque una diferencia en el rendimiento académico de estudiantes con los cuales trabajan. Esta perspectiva se explicaría por dos motivos: a) las condiciones de practicante en las que se encuentran, que no les permitiría libertad para trabajar (hasta cierto punto, ello es esperable como resultado) y b) las circunstancias previas de enseñanza en el establecimiento donde ejecutan la práctica, que no superarían las expectativas previas de los practicantes.

Es restringida la disponibilidad para realizar intervenciones con los estudiantes (M. T. Educación Diferencial, comunicación personal, 11 de junio de 2018).

Creo que los estudiantes no dominan contenidos previos y en las evaluaciones les va mal (D. C. Pedagogía en Matemáticas, comunicación personal, 11 de abril de 2018).

La segunda postura radica en la percepción de que las personas practicantes sí reconocen su influencia sobre los rendimientos académicos de estudiantes con los cuales trabajan. Esto se debería principalmente a un compromiso y dedicación con los logros y aprendizajes de sus estudiantes. Tal actitud destaca como algo que puede ir más allá de exigencias propias del establecimiento; específicamente, correspondería a actitudes y motivaciones de los propios practicantes.

A que con el tiempo he aprendido cómo aprenden los niños y las niñas y así he logrado aportar (V. G. Pedagogía Básica, comunicación personal, 16 de mayo de 2018).

Un grupo aprende más rápido que el otro, hay varios con dificultades, pero es en esos casos donde pongo mis conocimientos y motivación para que se den cuenta que son capaces de más (C. V. Educación Diferencial, comunicación personal, 28 de abril de 2018).

A que me preocupo de conocer como aprenden, que logros deben tener y como apoyarlos para obtenerlos, en ese sentido siempre trato de evitar que se frustren (V. R. Pedagogía en Lenguaje, comunicación personal, 10 de abril de 2018).

Otro factor que puede explicar el resultado anterior es que las personas practicantes no reconocen su influencia en el rendimiento académico de estudiantes con los que trabajan como un factor que, realmente, los diferencie de estudiantes de otras instituciones, independiente de si influyen significativamente o no. Para resolver estas interrogantes, será necesario un análisis estadístico de los rendimientos académicos de los establecimientos, análisis que se considera parte de una etapa posterior de investigaciones sobre identidad profesional.

\section{Sello de la UCSC y su influencia sobre la identidad profesional del practicante}

En esta investigación se identificaron cuatro características asociadas al sello que la UCSC busca transmitir a estudiantes. Para este propósito, se creó una escala Likert, basada en los focos y valores del Plan Estratégico Institucional de la UCSC, que buscaba medir hasta qué punto los practicantes consideran dichos aspectos del sello institucional como parte de su identidad profesional. Las afirmaciones de la escala y su respectiva asociación cualitativa son las siguientes:

1. En mi paso por la UCSC estoy desarrollando una formación integral que abarca aspectos tanto profesionales como de la ética humanista ( $85 \%$ de las respuestas sumando de acuerdo y muy de acuerdo).

Me motivo personalmente a ser un estudiante integral, aportando a mis estudiantes no solo en lo curricular, si tienen problemas personales o de otro tipo, tengo la cercanía como para apoyarlos en eso hasta donde pueda (D. Ch. Educación Diferencial, comunicación personal, 24 de abril de 2018).

2. En mi paso por la UCSC puedo reflexionar y desarrollar el respeto por la dignidad de la persona humana (86\% respuestas sumando de acuerdo y muy de acuerdo).

Desde primer año nos inculcan el respeto hacia los demás y tratar a los estudiantes y al resto de la comunidad como personas y que cada uno de ellos es igual e importante (J. A. Educación Diferencial, comunicación personal, 23 de abril de 2018). 
3. En mi paso por la UCSC pude notar en mi formación el sello de la universidad de servicio a la comunidad (73\% respuestas sumando de acuerdo y muy de acuerdo).

Por el compromiso en el ejercicio docente y el buen trato con la comunidad educativa (V. R. Pedagogía en Lenguaje, comunicación personal, 10 de abril de 2018).

4. Gracias a mi paso por la UCSC estoy desarrollando competencias pedagógicas efectivas ( $87 \%$ respuestas sumando de acuerdo y muy de acuerdo).

Las mallas de la UCSC, especialmente nuestra malla, se enfocan en potenciar estas habilidades a través de los ramos de didáctica o de educación (V. R. Pedagogía en Lenguaje, comunicación personal, 10 de abril de 2018)

Tengo un buen trato, respetuoso con ellos, trato de ayudarlos siempre, a no criticarlos siempre, a la confianza que perciben en mi persona, además trato de hacer mis clases más dinámicas y así participan más (V. C. Pedagogía en Educación Física, comunicación personal, 29 de junio de 2018)

Los datos tanto cualitativos como cuantitativos manifiestan, desde la visión de los practicantes, la construcción de su identidad profesional con base en componentes individuales (vocación personal) y componentes del sello institucional que la UCSC, efectivamente, desarrolla con sus estudiantes (formación en la UCSC).

\section{Valoración del practicante de su proceso de formación}

Mediante una escala Likert, los practicantes calificaron si, en su paso por la UCSC, han logrado desarrollar las siguientes dimensiones asociadas la vocación docente y el talento pedagógico (Castro y Jaramillo, 2018).

a) Compromiso social: la perspectiva del y la docente, como agente primordial del proceso educativo, define un compromiso profundo y permanente con sus alumnos y con su práctica, de manera de responder a lo que la realidad le demanda en favor de la formación (Bustamante, 2006, citado en Castro y Jaramillo, 2018, p.45). En este ítem, de acuerdo y muy de acuerdo agruparon el 91\% de las respuestas.

b) Motivación para enseñar: entendida como motivación intrínseca, cuando se involucra en la enseñanza por la satisfacción personal que le proporciona enseñar a otros, y motivación extrínseca, cuando la enseñanza se convierte en un fin; en este caso, la motivación es cumplir la jornada lectiva y los preceptos legales que justifican el trabajo docente (Ruiz, 2015, citado en Castro y Jaramillo, 2018, p.46). En este ítem, de acuerdo y muy de acuerdo agruparon el 93\% de las respuestas.

c) Rol profesional: profesional consciente de la transcendencia social de su trabajo y capaz de acometer sus obligaciones como agente educativo en cuyas manos descansa, en parte, la viabilidad de una educación que oficie como compensadora de las desigualdades sociales (Dueñas, 2009, citado en Castro y Jaramillo, 2018, p.47). En este ítem, de acuerdo y muy de acuerdo agruparon el 92\% de las respuestas.

d) Habilidades cognitivas: es el dominio destacado de habilidades, conocimientos o destrezas sistemáticamente desarrollados en, al menos, un campo de la actividad humana (Gagné, 2000, citado en Castro y Jaramillo, 2018, p.48). Se asocia al intelecto general, aptitudes académicas específicas, pensamiento creativo o productivo, habilidades de liderazgo, artes visuales o interpretativas y habilidades psicomotoras (Bralic y Romagnoli, 2000, citado en Castro y Jaramillo, 2018, p.48). En este ítem, de acuerdo y muy de acuerdo agruparon el $86 \%$ de las respuestas.

e) Habilidades sociales: conductas que expresan los sentimientos, actitudes, deseos, opiniones o derechos de ese individuo en un contexto interpersonal, pero, respetando las conductas de los demás individuos solucionando problemas presentes y reduciendo dificultades futuras (Caballo, 2007, citado en Castro y Jaramillo, 2018, p.49). En este ítem, de acuerdo y muy de acuerdo agruparon el 85\% de las respuestas.

Los resultados demostraron que la autopercepción de los practicantes sobre el desarrollo de estas dimensiones en su paso por la UCSC es positiva, considerando que, para las tres primeras dimensiones 
asociadas a vocación docente, las respuestas muy de acuerdo y de acuerdo suman más del $90 \%$ de las respuestas. La excepción se da en las dimensiones relacionadas con el talento pedagógico: habilidades cognitivas, con un $86 \%$ y habilidades sociales, con un $85 \%$ de respuestas con valoración muy de acuerdo y de acuerdo. En estas dimensiones, los resultados pueden explicarse por su asociación con el talento pedagógico, ya que la poca experiencia laboral podría traducirse en una autopercepción más prudente respecto del dominio de habilidades, conocimientos y destrezas.

Considerando los análisis cualitativos anteriores, la Tabla 2 permite observar una relación entre los resultados cualitativos y cuantitativos, asociados a los focos y valores que la UCSC busca transmitir a sus estudiantes.

TABLA 2

Cuadro asociación de resultados

\begin{tabular}{|l|l|l|}
\hline $\begin{array}{l}\text { Focos y } \\
\text { valores del } \\
\text { Plan }\end{array}$ & $\begin{array}{l}\text { Hallazgos del análisis } \\
\text { cualitativo }\end{array}$ & $\begin{array}{l}\text { Dimensiones de } \\
\text { la vocación y el } \\
\text { talento } \\
\text { pedagógico }\end{array}$ \\
$\begin{array}{l}\text { Institucional } \\
\text { de la Ucsc }\end{array}$ & & \\
\hline $\begin{array}{l}\text { Formación } \\
\text { integral de } \\
\text { personas }\end{array}$ & $\begin{array}{l}\text { - Preocupación por el y la } \\
\text { estudiante más allá de } \\
\text { cuestiones curriculares - }\end{array}$ & $\begin{array}{l}\text { Compromiso } \\
\text { social } \\
\text { Motivación para } \\
\text { enseñar Rol } \\
\text { profesional }\end{array}$ \\
\hline $\begin{array}{l}\text { Respeto por la } \\
\text { dignidad de la } \\
\text { persona } \\
\text { humana }\end{array}$ & $\begin{array}{l}\text { Costudiante como una } \\
\text { persona, que adquiere más } \\
\text { que contenidos }\end{array}$ & \\
\hline $\begin{array}{l}\text { Sello } \\
\text { institucional } \\
\text { servicio a la } \\
\text { comunidad }\end{array}$ & $\begin{array}{l}\text { - Buena relación, percepción } \\
\text { y adaptación del practicante } \\
\text { al establecimiento donde } \\
\text { desarrolla su práctica }\end{array}$ & $\begin{array}{l}\text { Compromiso } \\
\text { social }\end{array}$ \\
\hline $\begin{array}{l}\text { Competencias } \\
\text { desarrolladas } \\
\text { en el proceso } \\
\text { de formación }\end{array}$ & $\begin{array}{l}\text { - Cercanía con las y los } \\
\text { estudiantes dada su } \\
\text { preocupación más allá de } \\
\text { cuestiones curriculares - } \\
\text { Métodos y desarrollo de } \\
\text { clases dinámicas que buscan } \\
\text { integrar a estudiantes }\end{array}$ & $\begin{array}{l}\text { Habilidades } \\
\text { cognitivas } \\
\text { Habilidades } \\
\text { sociales }\end{array}$ \\
\hline
\end{tabular}

Fuente. Elaboración propia

Los hallazgos cualitativos, expresados en la preocupación por el y la estudiante más allá de cuestiones curriculares y su asociación con el comprender al estudiante como persona que no solo adquiere contenidos, se vinculan con hallazgos cuantitativos, en cuanto a la buena autoevaluación de estudiantes sobre su compromiso social, motivación para enseñar y su rol profesional. Este vínculo a su vez, se asocia con la formación integral de personas y el respeto por la dignidad de la persona humana, dicho de otra forma, estos valores que busca difundir la UCSC en sus estudiantes, se expresa a través de estos resultados.

El sello institucional de servicio a la comunidad, se expresa a través de la buena relación, percepción y adaptación del practicante al establecimiento donde desarrolla su práctica. Este resultado se asocia con la buena autoevaluación que realizan las y los estudiantes sobre su compromiso social.

Por último, las competencias desarrolladas en el proceso de formación, se vinculan a la cercanía de las y los estudiantes en práctica profesional, con estudiantes del establecimiento de práctica, dada su preocupación más allá de cuestiones curriculares. Dicha cercanía se traduce en métodos y el desarrollo de clases dinámicas que buscan integrar a estudiantes. Estos resultados cualitativos, relacionados entre sí, mediante un planteamiento causa efecto, es decir, la cercanía con estudiantes facilita el desarrollo de actividades pedagógicas, se asocia con los resultados cuantitativos referentes a las habilidades cognitivas y 
las habilidades sociales, implicando una buena autoevaluación en ambas habilidades, las cuales componen el talento pedagógico.

\section{Discusión TEÓRICA}

Los resultados corroboran la relevancia de las dimensiones identificadas en la revisión conceptual asociada a la relación entre docentes y estudiantes de los establecimientos donde desarrollan sus actividades pedagógicas (Czop, 2008; Gohier y Alin, 2000; Jiménez, 2015; Maroy, 2001; Vonck, 1995; Woods, 2002).

Esta relevancia se corrobora en la autoevaluación de las y los estudiantes, pues asocian la buena relación que perciben con los actores de la comunidad escolar (estudiantes y autoridades del establecimiento), con el desarrollo de actividades pedagógicas más efectivas. Esta asociación, es el resultado que da cuenta y responde al objetivo de esta investigación, pues en ella se plantea explícitamente la base de la identidad profesional descrita en este estudio.

Expresado el punto anterior, es necesario exponer los límites que tiene esta investigación, pues no se abordó en los análisis cuantitativos, los resultados académicos en los establecimientos, por lo tanto, es difícil afirmar con certeza, que los resultados anteriores efectivamente se traducen en una influencia positiva en el rendimiento. Este límite se explica en cuanto al contenido de los datos, pues se trabajan con autopercepciones expresadas en los discursos de estudiantes en práctica, sobre sus actividades pedagógicas, lo cual permitió abordar las emociones y percepciones que desarrollan (Mejía, 2013).

De esta forma, es pertinente plantear de manera prospectiva, la importancia de darle continuidad a esta investigación, abordando directamente un estudio cuantitativo de los rendimientos académicos, complementando los hallazgos cuantitativos y cualitativos de esta investigación.

Los hallazgos ciertamente manifiestan características que la UCSC buscó transmitir en sus estudiantes. Sin embargo, aun cuando consideran a la universidad como un factor relevante en el desarrollo de aspectos éticos y competencias para la actividad docente, esto no quiere decir que interpreten a la universidad como el único factor que les permitió desarrollar aspectos, sobre todo los éticos, pues se identificó la relevancia del rol de la universidad en un diálogo con las experiencias personales del estudiantado.

En síntesis, la identidad fue abordada desde tres enfoques: las identidades situacionales o transitorias (Barberousse y Vargas, 2016), pues el instrumento se enfocó en la influencia de los contextos de práctica profesional, donde las y los estudiantes construyen y socializan sus experiencias, desarrollando de esta forma una identidad flexible, adaptando las actividades docentes al contexto de los establecimientos. Precisamente esta flexibilidad, se evidencia en los aspectos vocacionales y expectativas personales de los practicantes, entendiendo este proceso desde las identidades profesionales asociadas a los procesos de enseñanza (Cattonar, 2001; Tardif, 2004).

Específicamente, la identidad profesional cada estudiante de la UCSC, corroboró la relevancia de los aspectos vocacionales y sus expectativas como futuro/a profesor/a, estableciendo como un factor relevante en las actividades académicas, la buena relación con estudiantes del establecimiento.

\section{Conclusiones}

Entendida como ir más allá del rol docente, la vocación personal refiere a aspectos personales del y la estudiante y la formación desarrollada en la UCSC, se expresa en la facilidad para llevar a cabo el aprendizaje; en su conjunto, estos son los factores que definen la identidad profesional de los practicantes, bajo la percepción de los actores con los cuales se trabajó en el estudio. En otros términos, la vocación permite mayor cercanía con estudiantes y esta cercanía repercute en facilidades para el aprendizaje. 
Los hallazgos cualitativos y el complemento generado por los resultados cuantitativos, permiten plantear hipótesis emergentes acerca de la identidad profesional del practicante de la UCSC:

1. Respecto de los valores y focos que la Universidad busca transmitir a sus estudiantes: efectivamente, se manifiestan expresiones sobre el vínculo que las personas practicantes establecen con sus estudiantes y cómo este vínculo influye en la forma en que desarrollan clases. Se propone, además, según los datos cualitativos y desde la percepción de los practicantes, un vínculo entre estas características identitarias y los rendimientos académicos de sus estudiantes de los establecimientos. Esto debe corroborarse en estudios posteriores mediante análisis estadísticos de los rendimientos académicos de los establecimientos.

2. Pese al carácter descriptivo de esta investigación, es posible establecer en los resultados cualitativos, asociaciones de tipo causa efecto: la UCSC se interpretó como un actor relevante (causa) en la formación (focos y valores de la UCSC) y la vocación personal. En su conjunto, estos aspectos fortalecen el vínculo con estudiantes de la comunidad escolar. Es decir, se identifican factores inherentes a la vocación personal y, además, factores propios de la influencia de la UCSC. Sin embargo, es necesario para la institución reflexionar, a la luz de estos datos, respecto de los mecanismos con los que transmite o fomenta aspectos éticos y cuál es la posición que puede asumir respecto de otros factores que influyen en el estudiantado en este aspecto.

3. Efectivamente, en las personas practicantes se logran interpretar factores que les diferencian de otros estudiantes de pedagogía de otras casas de estudio, hecho el cual corrobora la existencia de una identidad profesional. Lo anterior implica un desafío para la institución, pues este resultado motiva a la reflexión respecto de la forma en que se construye esta identidad, específicamente como esta identidad tiene coherencia respecto del perfil de egreso de las carreras de la facultad de educación y de qué manera potenciar las características de la identidad de sus estudiantes.

Los factores vocación personal y la formación desarrollada en la UCSC son los componentes centrales de la caracterización. Así, es posible dar respuesta al objetivo general de este estudio y corroborar el supuesto de investigación, definido a partir del marco conceptual.

\section{REFERENCIAS}

Barberousse, P., y Vargas, M. (2016). Orientaciones internacionales y regionalespara la formación docente. Un entramado pertinente al diseño de planes de estudio y los procesos formativos docentes iniciales: el caso de la DEB-CIDE-UNA en Costa Rica. En A. Cannellotto (Presidencia) Simposio llevado a cabo en el VII Seminario Internacional: Valorización de la Profesión y la Formación Docente- Red KIPUS, Buenos Aires, Argentina.

Beijard, D., Meijer, P. y Verloop, N. (2004). Reconsidering research on teachers' profesional identity. Teaching and Teaching Education 20, 107-128.

Bericat, E. (1998). La integración de los métodos cuantitativo y cualitativo en la investigación social. España: Editorial Ariel.

Blanco, N. y Pirela, J. (2016). La complementariedad metodológica: Estrategia de integración de enfoques en la investigación social, Espacios Públicos, 19(45), 1-2. Recuperado de https://bit.ly/2sJT2ap

Camacho, X., Rojas, M. y Hernández, L. (2019). La identidad con el entorno rural en la superación profesional del director de primaria. Revista Atenas, 1(45), 1-2. Recuperado de https://www.redalyc.org/articulo.oa?id=4780 /478058273010

Canquiz, L y Maldonado, M. (2016). Evaluación de competencias docentes en el desarrollo de la práctica pedagógica. Omnia, 22(2), 37-49.

Cattonar, B. (2001). Les identités professionnelles enseignantes. ébauche d'un cadre d'analyse. Cabiers de Recherche du Girsef, 10, 1-34. 
Castro, R. y Jaramillo, C. (2018). Autopercepción de estudiantes novatos de pedagogías relativa a la vocación y talento pedagógico. Revista Reflexión E Investigación Educacional, 1(1), 33-56. Recuperado de http://revistas.ubiobio.c 1/index.php/REINED/article/view/3404

Cervantes, E. y Gutiérrez, P. (2013). Identidad y trabajo docente: necesidades de formación de las y los maestros en Ciudad Juárez. Revista Atenas, 4(24), 1-12 Recuperado de https://www.redalyc.org/pdf/4780/478048960004 .pdf

Czop, L. (2008). Professional identity of a reading teacher: responding to high-stakes testing pressures. Teachers and teaching: theory and practice, 14(3), 239-252.

De Laurentis, C. (2015). Identidad Docente: Herramientas para una aproximación narrativa. Revista EntramadosEducación y Sociedad, 2(2), 67-74.

Galaz, A. (2011). El profesor y su identidad profesional ¿facilitadores u obstáculos del cambio educativo? Estudios Pedagógicos, 37(2), 89-107.

Gohier, C. y Alin, C. (2000). Enseignant-Formateur. La construction de l'Identité professionnelle. Recherche et Formation. Paris: L'Harmattan.

González, E. (2017). Formación docente y construcción de identidad profesional. Análisis desde la historia reciente de la Escuela Normal Juan Demóstenes Arosemena (tesis doctoral). Universidad Especializada de las Américas, Panamá.

Jiménez, E. (2015). Desarrollo personal y profesional de maestras de educación primaria: aportes de la orientación. Revista Electrónica Actualidades Investigativas en Educación, 15(1), 1-29.

Kronenberg, F. y Ramugondo, E. (2013). Explaining Collective Occupations from a Human Relations Perspective: Bridging the Individual-Collective Dichotomy.Journal of Occupational Science, 22(1), 3-16. Recuperado de htt ps://www.tandfonline.com/doi/abs/10.1080/14427591.2013.781920

Lizana, V. (2009). Una Relación Invisibilizada en los Contextos de Formación Docente Inicial: La identidad Profesional desde una Perspectiva de Género. Revista Iberoamericana sobre Calidad, Eficacia y Cambio en Educación, 7(3), 69-81.

López, P. y Fachelli, S. (2015). Metodología de la investigación social cuantitativa. Bellaterra, España: Universitat Autònoma de Barcelona. Recuperado de https://ddd.uab.cat/record/129382

Maroy, C. (2001). Le modèle du praticien réflexif à l'épreuve de l'enquête. Les Cabiers de Recherche du Girsef, 12, 2-26.

Mejía, J. (2013). Problemas del conocimiento en ciencias humanas. La cuestión del método y el proyecto de investigación cualitativa. Investigación Cualitativa, 17(2), 27-47.

Miranda, G. y Vargas, M. (2019). Identidad profesional y formación docente universitaria: Un proceso en construcción desde la mirada del estudiantado. Revista Electrónica Actualidades Investigativas en Educación, 19(1), 1-18.

Modelo Educativo de la Universidad Católica de la Santísima Concepción. (2017). Chile. Recuperado de https://ww w.ucsc.cl/wp-content/uploads/2017/07/MODELO_EDUCATIVO_MARZO2017_web.pdf

Morales, Y. (2016). Análisis de la reflexión presente en las crónicas de estudiantes en formación inicial en Educación Matemática durante su periodo de práctica profesional. Acta Scientiae Canoas, 19(1),122-137.

Programa de Mejoramiento Institucional USC 1501 de la Universidad Católica de la Santísima Concepción [PMI USC 1501]. (2015). Chile. Recuperado de https://www.ucsc.cl/wp-content/uploads/2014/05/objetivo-gener al-especifico.pdf

Schick, C., Galaz, A. y Urrutia, D. (2015). Identidad profesional y factores de riesgo ocupacional de las profesoras. Estudios Feministas, 23(3), 803-815.

Strauss, A. y Corbin, J. (2002). Bases de la investigación cualitativa. Técnicas y procedimientos para desarrollar la teoria fundamentada. Contus, Colombia: Universidad de Antioquía.

Tardif, M. (2004). Los saberes del docente y su desarrollo profesional. Madrid: Narcea.

Trainer, J. (2013). Pensar la identidad institucional en contextos contemporáneos: sobre relatos, modelos, metáforas y abordajes. Rosario, Argentina, en los umbrales del Siglo XXI. Revista Educación, 37(1), 161-178.

Vieytes, R. (2004). Metodología de la Investigación en organizaciones, mercado y sociedad. Buenos Aires: Editorial de las Ciencias. 
Vonck, H. (1995). Teacher induction: an essential elemental at the start of teachers. Revista Española de Pedagogía, 53(200), 67-91.

Woods, P. (2002). The reconstruction of primary teachers' identities. British Journal of Sociology of Education, 23(1), 89-106.

\section{Notas}

1 La UCSC para efectos de esta investigación

\section{BY-NC-ND}

